\title{
The significance of cysteine synthesis for acclimation to high light conditions
}

\section{Anna Speiser ${ }^{1}$, Stefan Haberland ${ }^{1}$, Mutsumi Watanabe ${ }^{2}$, Markus Wirtz $^{1}$, Karl-Josef Dietz ${ }^{3}$, Kazuki Saito ${ }^{4,5}$ and Rüdiger Hell ${ }^{1}$ *}

1 Plant Molecular Biology, Centre for Organismal Studies, University of Heidelberg, Heidelberg, Germany

${ }^{2}$ Molecular Plant Physiology, Max Planck Institute of Molecular Plant Physiology, Potsdam, Germany

${ }^{3}$ Plant Biochemistry and Physiology, University of Bielefeld, Bielefeld, Germany

${ }^{4}$ RIKEN Center for Sustainable Resource Science, Yokohama, Japan

${ }^{5}$ Graduate School of Pharmaceutical Sciences, Chiba University, Chiba, Japan

\section{Edited by:}

Stanislav Kopriva, University of Cologne, Germany

Reviewed by:

Uener Kolukisaoglu, University of

Tuebingen, Germany

Luis C. Romero, Consejo Superior

de Investigaciones Científicas, Spain

*Correspondence:

Rüdiger Hell, Plant Molecular

Biology, Centre for Organismal

Studies, Im Neuenheimer Feld 360,

69115 Heidelberg, Germany

e-mail: ruediger.hell@

cos.uni-heidelberg.de
Situations of excess light intensity are known to result in the emergence of reactive oxygen species that originate from the electron transport chain in chloroplasts. The redox state of glutathione and its biosynthesis contribute importantly to the plant's response to this stress. In this study we analyzed the significance of cysteine synthesis for long-term acclimation to high light conditions in Arabidopsis thaliana. Emphasis was put on the rate-limiting step of cysteine synthesis, the formation of the precursor O-acetylserine (OAS) that is catalyzed by serine acetyltransferase (SERAT). Wild type Arabidopsis plants responded to the high light condition $\left(800 \mu \mathrm{mol} \mathrm{m} \mathrm{m}^{-2} \mathrm{~s}^{-1}\right.$ for 10 days) with synthesis of photo-protective anthocyanins, induction of total SERAT activity and elevated glutathione levels when compared to the control condition $\left(100 \mu \mathrm{mol} \mathrm{m} \mathrm{m}^{-2} \mathrm{~s}^{-1}\right)$. The role of cysteine synthesis in chloroplasts was probed in mutant plants lacking the chloroplast isoform SERAT2;1 (serat2;1) and two knock-out alleles of CYP20-3, a positive interactor of SERAT in the chloroplast. Acclimation to high light resulted in a smaller growth enhancement than wild type in the serat2;1 and cyp20-3 mutants, less induction of total SERAT activity and OAS levels but similar cysteine and glutathione concentrations. Expression analysis revealed no increase in mRNA of the chloroplast SERAT2;1 encoding SERAT2;1 gene but up to 4.4-fold elevated SERAT2;2 mRNA levels for the mitochondrial SERAT isoform. Thus, lack of chloroplast SERAT2;1 activity or its activation by CYP20-3 prevents the full growth response to high light conditions, but the enhanced demand for glutathione is likely mediated by synthesis of OAS in the mitochondria. In conclusion, cysteine synthesis in the chloroplast is important for performance but is dispensable for survival under long-term exposure to high light and can be partially complemented by cysteine synthesis in mitochondria.

Keywords: high light stress, SERAT, CYP20-3, chloroplasts, mitochondria, glutathione

\section{INTRODUCTION}

Reactive oxygen species (ROS) play a dual role in plants since they function as regulators and if excessively produced as harmful reactive metabolites. Protein thiols are particularly sensitive to redox regulation, but also to oxidative damage by ROS (Meyer and Hell, 2005). Formation of ROS continuously occurs at low rates during photosynthetic electron transport. However, exposure of plants to high light stimulates release of ROS such as singlet oxygen, superoxide and hydrogen peroxide from the electron transport chain and may cause oxidative stress (Barber and Andersson, 1992; Fryer et al., 2002). The effects on growth of high light and the associated excess excitation energy in chloroplasts have been discussed in literature extensively (Foyer et al., 2009; Suzuki et al., 2012; Szechyńska-Hebda and Karpiński, 2013). Among these responses are morphological changes of the leaves (Eckardt et al., 1997), accumulation of anthocyanins as scavengers of evolving ROS (Chalker-Scott, 1999; Gould et al., 2002; Vanderauwera et al., 2005; Zeng et al., 2010) and the expression of high light-induced genes (Alsharafa et al., 2014). A well-characterized excess light-induced gene is APX2 that encodes for ascorbate-peroxidase 2, as part of the ascorbate-glutathione ROS detoxification cycle (Rossel et al., 2006; Foyer and Noctor, 2011; Noctor et al., 2011).

Reduced glutathione and its oxidized form represent the most abundant low-molecular weight thiol redox couple found in eukaryotes and prokaryotes and plays a crucial role in adjustment of cellular redox potential and signaling of ROS stress (May et al., 1998; Noctor and Foyer, 1998; Rouhier et al., 2008). Changes of the ratio of reduced to oxidized glutathione and gene expression and activity of glutathione reductase (GR) in response to oxidative stress have frequently been reported (Noctor et al., 2011; Chan et al., 2013). Reduction of oxidized glutathione 
takes place in the plastids, mitochondria, cytosol and peroxisomes and is so essential for survival of plants that even back-up systems have evolved by NADPH-dependent thioredoxin reductases (Marty et al., 2009). Increases of the steady-state level of the glutathione pool in response to different light conditions are reportedly relatively small (Noctor et al., 2012), but excess oxidized glutathione has been hypothesized to be removed from the cytosolic pool and directed to the vacuole for degradation by $\gamma$-glutamyltransferase to recycle cysteine (Grzam et al., 2007; Noctor et al., 2012).

The functional component of glutathione is cysteine that is synthesized from sulfide, the endproduct of the reductive sulfate assimilation pathway in plastids, and from $O$-acetylserine (OAS) by the enzyme O-acetylserine (thiol) lyase (OAS-TL). OAS is synthesized from serine and acetyl coenzyme A by serine acetyltransferase (SERAT) whose activity limits the overall rate of cysteine synthesis (Hell and Wirtz, 2011). In Arabidopsis thaliana the SERAT protein family is encoded by five isoforms that localize to the cytosol, the mitochondria and the plastids. In unstressed leaves of Arabidopsis the major source of OAS synthesis (79\%) are the mitochondria (SERAT2;2) (Haas et al., 2008; Watanabe et al., 2008b), whereas cytosolic SERAT1;1 and chloroplastidic SERAT2;1 contribute 9 and 13\%, respectively, of total SERAT activity (Watanabe et al., 2008b). Transcriptional changes in SERAT expression levels have several times been observed. Howarth et al. (2003) reported kinetic changes in mRNA abundance of all three major SERAT genes in response to cadmium exposure in leaf and root. SERAT2;2 and SERAT2;1 transcripts were increased in roots by treatment with the oxidizing reagent menadione (Lehmann et al., 2009). Transfer of a catalase-deficient mutant (cat2) from high $\mathrm{CO}_{2}$ concentration to a normal environment resulted in a strong (up to 10-fold) induction of plastidic SERAT2;1 transcript levels (Queval et al., 2009). Correspondingly, public gene expression databases show up to 15 -fold elevations of SERAT2; 1 expression upon $\mathrm{H}_{2} \mathrm{O}_{2}$ treatments (Genevestigator ${ }^{\circledR}$ V3; Hruz et al., 2008; https:/genevestigator.com/gv/plant.jsp). With respect to transcription levels and specific activities the cytosolic isoforms SERAT3;1 and SERAT3;2 are considered to be the minor isoforms (reviewed in Hell and Wirtz, 2011).

SERAT activity in the three compartments of cysteine synthesis is modulated in vivo by two post-transcriptional mechanisms. In the first mechanism, SERAT and OAS-TL proteins reversibly interact with each other and form the hetero-oligomeric cysteine synthase complex (CSC). Formation and dissociation of the complex constitute a metabolic regulatory model, positioning the CSC as a sensor for cellular sulfur homeostasis (Hell and Wirtz, 2011). Sulfide stabilizes the complex and prevents SERAT from feedback inhibition by cysteine (Wirtz et al., 2012) whereas in the absence of sulfide high OAS concentrations dissociate the complex and thereby adjusting SERAT activity and thus OAS production depending on the actual sulfide supply (Hell and Wirtz, 2011). In the second mechanism SERAT2; 1 is postulated to be activated by CYP20-3 mediated CSC association in the stroma (Dominguez-Solis et al., 2008; Park et al., 2013).

Cyclophilins (CYPs) belong to the superfamily of immunophilins, also including FK506- and rapamycinbinding proteins (FKBPs). Both groups of proteins harbor a peptidyl-prolyl cis/trans isomerase activity, favoring and accelerating the cis/trans transition of peptidyl-prolyl bonds during folding and multimerization of proteins and thus comprising foldase- and chaperone-like functions (Wang and Heitman, 2005). In the Arabidopsis genome 52 immunophilin-encoding genes were identified of which 23 are putative FKBPs and 29 are putative CYP proteins (He et al., 2004; Romano et al., 2004). Subcellular localization studies reveal that 13 CYPs are in the cytosol (Chou and Gasser, 1997), three CYPs are present in the nucleus (Romano et al., 2004), and two CYPs harbor a mitochondrial localization motif. Arabidopsis contains further six genes encoding plastidic CYPs of which only one isoform localizes to the stroma (CYP20-3) (Lippuner et al., 1994), whereas the remaining five CYPs are targeted to the thylakoid lumen. CYP203 is the sixth most abundant protein in the stroma (Lippuner et al., 1994; Peltier et al., 2006), suggesting multiple targets for its foldase and chaperone activities. It contains two internal disulfide bonds and its activity was shown to be dependent on thioredoxin-triggered and thus redox-related conformational changes (Motohashi et al., 2003; Laxa et al., 2007).

Investigations of the Arabidopsis cyp20-3 (= roc4; rotamase CYP 4) mutant found a growth retardation under continuous elevated light exposure (up to $1000 \mu \mathrm{mol} \mathrm{m} \mathrm{m}^{-2} \mathrm{~s}^{-1}$ ) and attributed one CYP20-3 function to the repair of photodamaged photosystem II (Cai et al., 2008). Enhanced light intensity (up to $300 \mu \mathrm{mol}$ $\mathrm{m}^{-2} \mathrm{~s}^{-1}$ ) and other ROS-inducing conditions (Dominguez-Solis et al., 2008) caused significant growth reduction of the same cyp20-3 mutant line (Dominguez-Solis et al., 2008). CYP20-3 was shown to interact with SERAT2;1, suggesting some sort of activation or stabilization of the enzyme or the cysteine synthase complex. A mechanism was proposed in which the chloroplast 2-Cys peroxiredoxin may oxidize CYP20-3 and photoreduced thioredoxin then reduces and activates CYP20-3, which in turn would promote cysteine synthesis by its protein-folding capabilities (Dominguez-Solis et al., 2008). This model was extended based on the binding of 12-oxo-phytodienoic acid (OPDA), the precursor of jasmonic acid with independent hormone function, to CYP20-3. Facilitated interaction of CYP20-3 to SERAT2;1 by OPDA was concluded as a key step in hormonal signaling toward cellular redox homeostasis in stress responses (Park et al., 2013).

In this study we investigated whether cysteine synthesis in the chloroplast has a specific role for high light acclimation in Arabidopsis, since it provides the thiol-harboring building block for glutathione. Reasoning that the lack of SERAT2;1 protein in plastids of a null mutant should result in similar or even more severe stress phenotypes compared to CYP20-3 loss of function mutants, we analyzed growth patterns, stress symptoms, cysteinerelated metabolites and SERAT gene expression. Thus, this study addresses the important question of stress-related redox homeostasis in context of site-specific activity of cysteine synthase complexes.

\section{RESULTS}

GENOMIC CHARACTERIZATION OF cyp20-3 T-DNA INSERTION LINES

Two different T-DNA insertion lines of the CYP20-3 gene as well as one of the serat2; 1 mutant, lacking plastidic SERAT (Watanabe et al., 2008b) were chosen to examine the effects of high light 


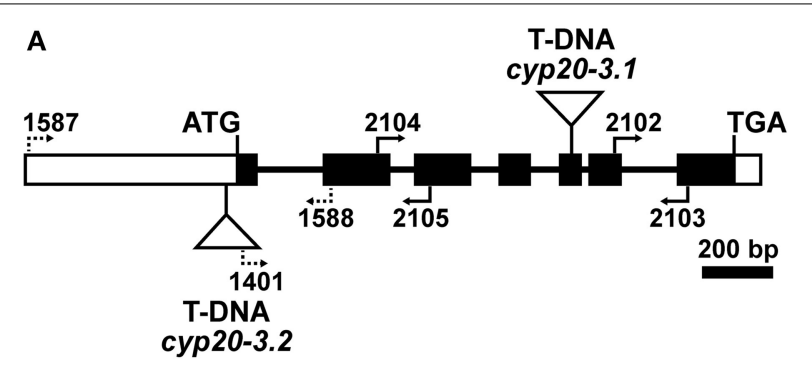

B

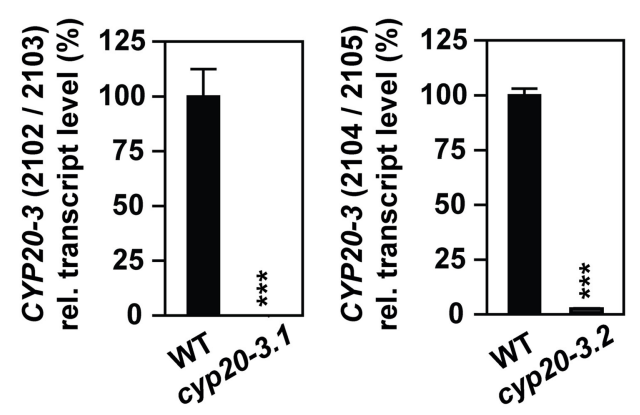

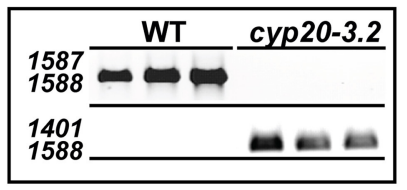

C

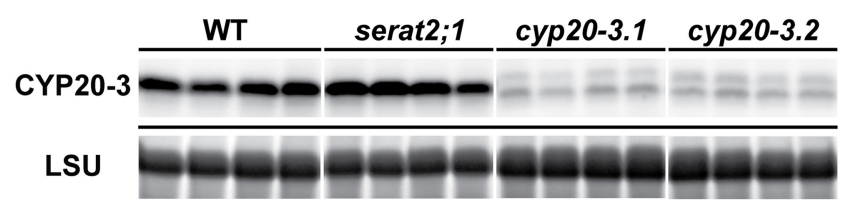

FIGURE 1 | Identification, molecular characterization and gene expression analysis of cyp20-3 mutant lines. (A) Gene model of the Arabidopsis thaliana gene CYP20-3 (AT3G62030). Exons and introns are illustrated as black boxes and thin lines, respectively. White boxes represent $5^{\prime}$ - and $3^{\prime}$-UTRs. Triangles define the two T-DNA insertions of cyp20-3.1 and cyp20-3.2, respectively. Black arrows and numbering indicate the positions of used primers. Molecular verification of cyp20-3.2 by PCR using specific primer combinations for the wild type (WT) allele (upper panel) and mutant allele (lower panel). (B) CYP20-3 gene expression levels by qRT-PCR with cyp20-3.1 and cyp20-3.2 cDNA and indicated primer combinations. WT transcript levels were set to $100 \%$. Statistically significant differences are indicated as asterisks (*** $p \leq 0.001$; Student's $t$-test). Results represent means $\pm S E(n=3)$. (C) Immunological detection of CYP20-3 using a polyclonal antibody against Arabidopsis CYP20-3 in leaf protein extracts of WT, serat2;1, cyp20-3.1 and cyp20-3.2 plants (four lanes each) grown under control conditions. Staining intensities of the large subunit of ribulose-1,5-bisphosphate carboxylase/oxygenase (LSU) protein in the same samples confirm equal loading in the individual lanes. on growth and cysteine synthesis. T-DNA positions and primer binding sites which were used for the molecular characterization of both cyp20-3 mutants are depicted in Figure 1A. One mutant line (SALK_001615) harbors a T-DNA insertion in the fifth exon and had been previously described as a full knockout of the expression of the CYP20-3 gene (Cai et al., 2008; Dominguez-Solis et al., 2008). In these studies this mutant allele was named cyp20-3 or roc4 and will herein be referred to as cyp20-3.1. Quantitative expression analysis of the CYP20-3 transcript in cyp20-3.1 was performed with qRT-PCR (Figure 1B) using $3^{\prime}$ nested primers and demonstrated a minimal expression level of $0.02 \%$ of the CYP20-3 transcript compared to wild type. A so far uncharacterized second T-DNA insertion mutant allele of CYP20-3, cyp20-3.2 was selected from the SALKcollection (SALK_054125) with the T-DNA residing upstream of the first exon in the $5^{\prime}$-UTR (Figure 1A). Homozygous plants were isolated based on PCR with specified primer combinations (Figure 1A). Transcript analysis with qRT-PCR in homozygous cyp20-3.2 mutant lines revealed a residual CYP20-3 expression level of $2.5 \%$ compared to wild type plants (Figure 1B). A polyclonal antibody against Arabidopsis CYP20-3 detected residual amounts of CYP20-3 in cyp20-3.1 and cyp20-3.2 that corresponded to the detected remaining mRNA levels (Figure 1C). It should be noted that no CYP20-3 protein was detected in cyp203.1 using a different antibody in an earlier study (Cai et al., 2008). Thus, both alleles are strong knock-down mutants of the cyp20-3 gene. The CYP20-3 protein level was unchanged in the serat2;1 mutant compared to wild type, excluding any compensatory upregulation in response to the lack of this interaction target.

\section{ACCLIMATION TO LONG-TERM EXPOSURE TO HIGH LIGHT CONDITIONS}

The effect of high light on Arabidopsis mutants with eliminated plastidic SERAT activity (serat2;1) or strongly depleted CYP20-3 expression (cyp20-3.1 and cyp20-3.2), was analyzed with plants that were grown under short-day conditions at a light intensity of $100 \mu \mathrm{mol} \mathrm{m} \mathrm{m}^{-2} \mathrm{~s}^{-1}$ until the age of 3 weeks and subsequently challenged with long-day light of $800 \mu \mathrm{mol} \mathrm{m}^{-2} \mathrm{~s}^{-1}$ irradiance (high light) for additional 10 days. Under control conditions with $100 \mu \mathrm{mol} \mathrm{m} \mathrm{m}^{-2} \mathrm{~s}^{-1}$ the growth phenotype of the mutant lines was similar to wild type plants (Figure 2A). Growth under high light conditions caused a decrease in rosette diameter and downward curling of leaves in wild type, serat2;1, cyp20-3.1, and cyp20-3.2 plants when compared to the phenotype of control plants. The mutant genotypes were affected in the same manner as the wild type plants, i.e., also the two cyp20-3 lines did not differ with respect to phenotypes unlike earlier reports (Cai et al., 2008; Dominguez-Solis et al., 2008). Protein levels of CYP20-3 were neither responding in wild type plants under high light nor in the serat2;1 mutant. The very low levels of CYP203 in cyp20-3.1 and cyp20-3.2 plants remained also unchanged (Supplementary Figure 1). Fresh weight determination revealed a 2.2-fold increase in biomass of high light-treated wild type plants compared to control wild type plants (Figure 2B). The fresh weight biomass of serat2;1, cyp20-3.1, and cyp20-3.2 increased 
only 1.7-, 1.6-, and 2.1-fold, respectively, when grown under high light (Figure 2B). Dry weight measurements of whole rosettes also showed a significant increase of biomass in all genotypes upon high light exposure (Figure 2C). Wild type plants exhibited a 3.5-fold increase, serat2;1 and cyp20-3.1 a 2.7-fold increase and cyp20-3.2 a 4.7-fold increase of dry weight upon high light treatment (Figure 2C).

Along with the stunted growth phenotype red leaf coloring was observed for the high light-treated plants at the abaxial side of the leaves (Supplementary Figure 2). Therefore, anthocyanin levels were determined on the basis of fresh weight (Figure 2D). Significant 2.3-fold to 3.1-fold increases in anthocyanin content could be observed for the wild type, serat2;1 and both cyp20-3 mutant lines when compared to anthocyanin levels of unstressed rosette leaves (Figure 2D). Noteworthy, the anthocyanin levels were indistinguishable between wild type and mutant lines, indicating that this stress response is independent of the presence of CYP20-3 or SERAT2;1. Thus, the applied high light conditions had two components: significant stress but also promoted growth. However, in comparison to wild type the serat2;1 and cyp20-3.1 and cyp20-3.2 mutants grew slower under high light conditions. Apparently activation of chloroplast SERAT2;1 by CYP20-3 as well as the presence of SERAT2;1 and thus the chloroplast synthase complex are dispensable, but both systems enhance the performance of Arabidopsis plants under highlight.

\section{IMPACT OF HIGH LIGHT ON CYSTEINE METABOLISM}

To test whether the observed stress susceptibility phenotype of the mutant lines was due to alterations of cysteine metabolism the total SERAT activity and steady-state OAS levels of leaves were determined. Both parameters are established biochemical markers for the state of cysteine synthesis (Haas et al., 2008; Heeg et al., 2008; Watanabe et al., 2008b; Khan et al., 2010). Compared to control conditions a 1.9 -fold increase of SERAT activity was observed in high light-treated wild type plants (Figure 3A). A smaller, but still significant increase under high light was also observed for serat2;1 (1.5-fold) and cyp20-3.2 (1.5-fold) mutants. SERAT activity was also 1.3-fold elevated in cyp20-3.1, but was found at the limit of statistical significance. Higher SERAT activity may result in higher OAS contents. Indeed, changes in OAS concentrations were in agreement with the increased SERAT activity (Figure 3B). A 2.6-fold increase in OAS content was observed for wild type plants exposed to high light conditions. In serat2;1, cyp20.3.1 and cyp20-3.2 OAS steady state levels increased 1.7-, 1.6-, and 2-fold, respectively. In accordance with the SERAT activity the raise of OAS concentrations upon high light treatment was smaller in the mutant plants than in the wild type plants. Statistical examination of SERAT activity and OAS content under control conditions revealed no difference between wild type and all three mutant lines. However, the SERAT activities and OAS concentrations in the three mutant lines increased significantly less under high light than those in wild type plants. The weak statistical significance $(p=0.106)$ of the difference of SERAT activity in cyp20-3.2 to wild type under high light might be owed to the leaky insertion allele and the higher residual CYP20-3 level of this mutant. The same consideration applies to the OAS concentration under high light in cyp20-3.2 $(p=0.120)$. Thus,

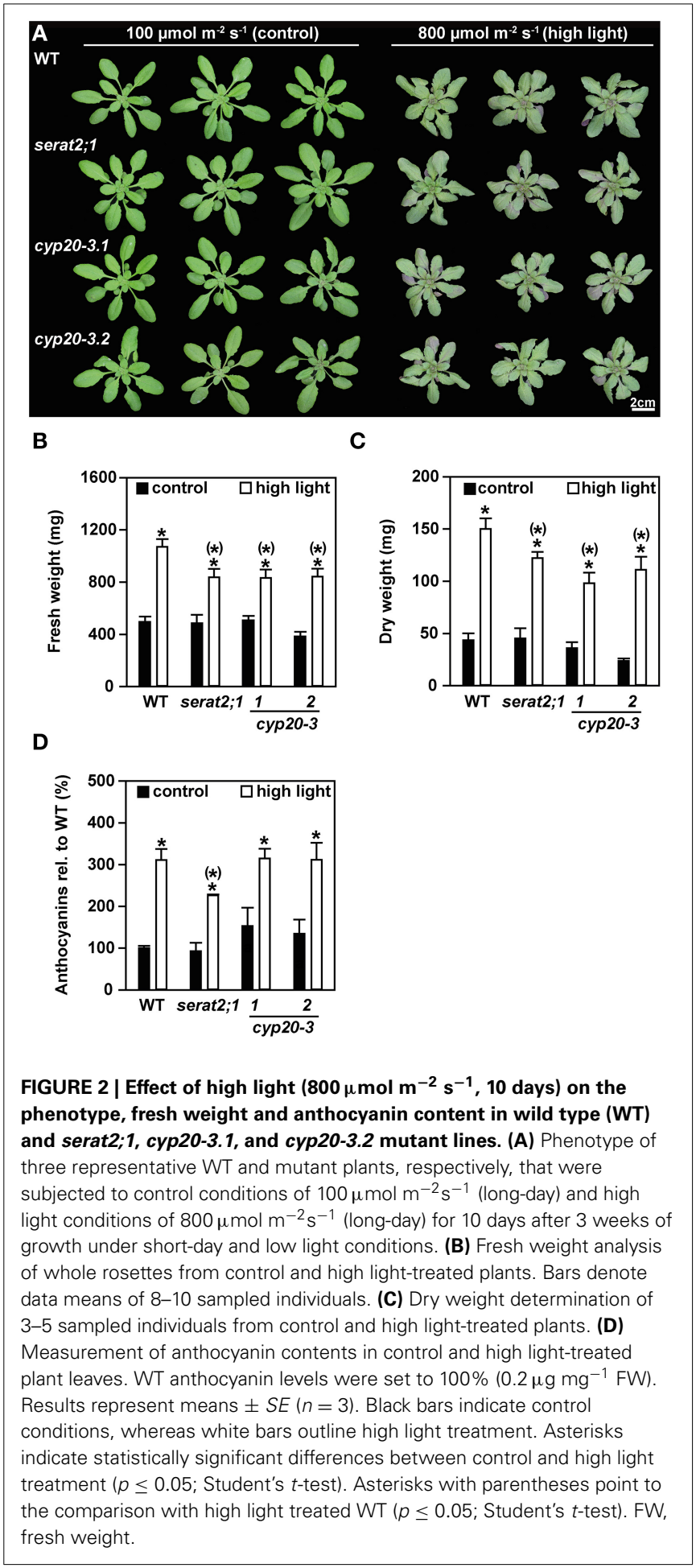

plastidic SERAT2;1 and its presumed activation by CYP20-3 is required for fully enhanced OAS synthesis, but about half of the observed increases of SERAT activity and OAS concentrations is likely due to events in mitochondria and cytosol.

Cysteine steady state levels were not significantly altered in high light-exposed wild type, serat2;1, cyp20-3.1 and cyp20-3.2 


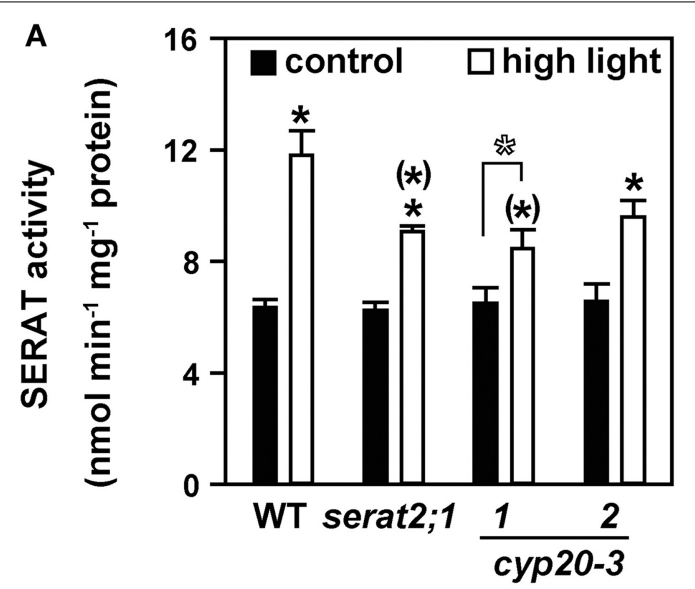

B

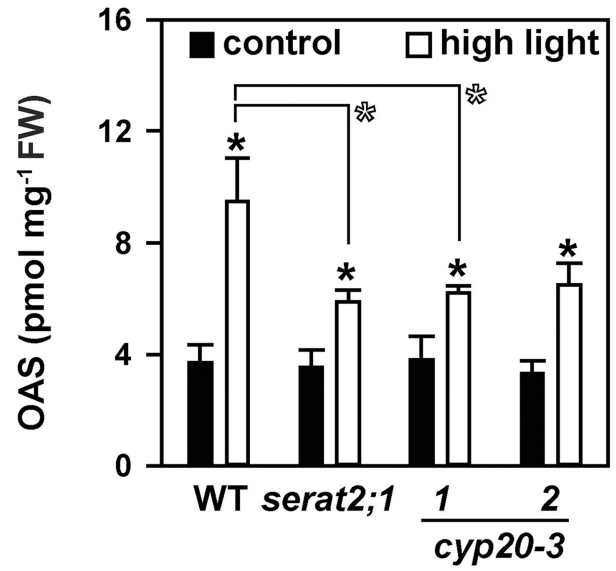

FIGURE 3 | Effect of 10 days high light treatment on SERAT activity and OAS steady state concentrations in wild type (WT) and serat2;1, cyp20-3.1, and cyp20-3.2 mutant lines. (A) Total SERAT activity was measured in leaf protein extracts of control and high light-treated WT and mutant plants $(n=3)$. (B) OAS concentrations were determined from leaf metabolite extracts of control and light-stressed plants $(n=5)$. Black bars indicate control conditions, whereas white bars outline stress conditions. Results represent means $\pm S E$. Statistical significances are based on Student's $t$-test. Differences between control and high light treatment are marked with asterisks ( $p \leq 0.05$ ). Differences between high light treated WT and mutants are marked by asterisks in parentheses $(p \leq 0.05)$. Open asterisks refer to indicated pairwise comparisons with $(p \leq 0.1)$. FW, fresh weight.

plants compared to unstressed plants (Figure 4A). Glutathione content was moderately but significantly increased in wild type (1.3-fold) upon high light exposure (Figure 4B) similar to previous reports (e.g., Alsharafa et al., 2014). In serat2;1 (1.1fold) and cyp20-3.2 (1.2-fold) the increase was lower than in wild type and found to be unchanged for cyp20-3.1. Cysteine and glutathione levels from all three mutant lines were not different from wild type levels under both conditions (Figure 4). Taken together, glutathione steady state levels increase as part of the acclimation response but were similar between wild type and mutants. This steady-state level determination indicates that OAS synthesis is enhanced and, while keeping cysteine levels unchanged, allows for increased glutathione concentrations under high light conditions.
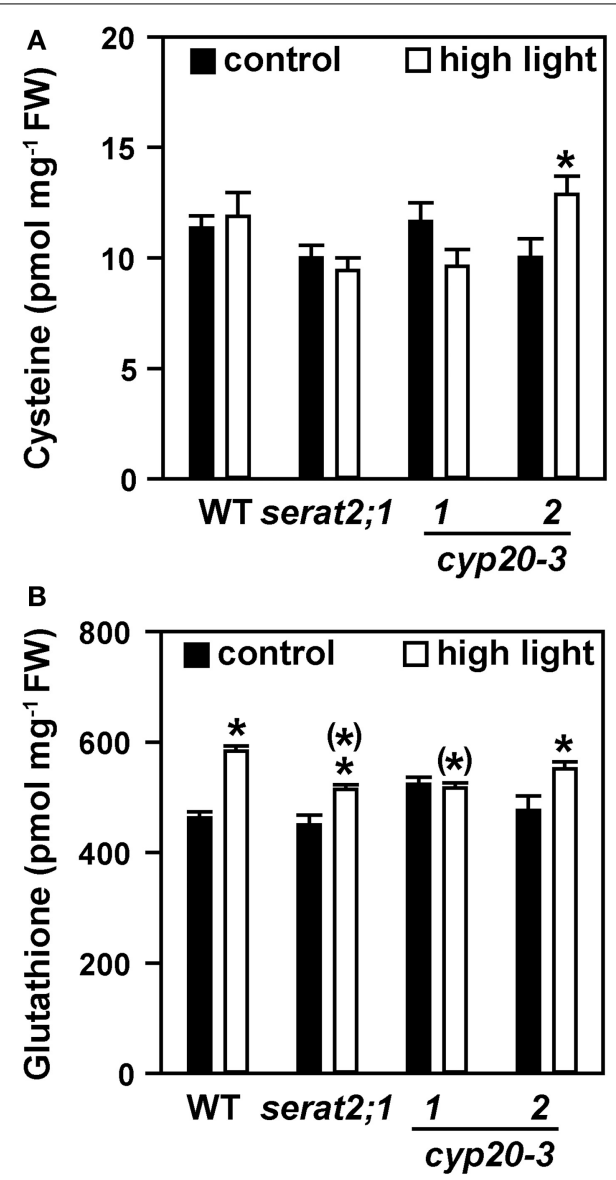

FIGURE 4 | Effect of 10 days high light exposure on leaf cysteine and glutathione steady state concentrations in wild type (WT) and serat2;1, cyp20-3.1, and cyp20-3.2 mutant lines. (A) Cysteine concentrations were determined from metabolite extracts of control and high light-treated WT and mutant plants. (B) Glutathione concentrations. Black bars indicate control conditions, whereas white bars outline stress conditions. Results represent means $\pm S E(n=5)$. Asterisks indicate statistically significant differences between control and high light treatment ( $p \leq 0.05$; Student's $t$-test). Asterisks in parentheses point to the comparison with high light-treated WT ( $p \leq 0.05$; Student's $t$-test). FW, fresh weight.

\section{IMPACT OF HIGH LIGHT ON SERAT TRANSCRIPT LEVELS}

The increase in total SERAT activity and OAS contents upon exposure to high light prompted us to determine the transcript levels of the three major SERAT isoforms. Transcript levels of the plastidic SERAT isoform (SERAT2;1) were indistinguishable between control and stressed plants in wild type, cyp20-3.1 and cyp20-3.2 lines (Figure 5A). serat2;1 RNA was not tested in this respect, as this line was already shown to be a full gene knockout mutant (Watanabe et al., 2008b). A significant increase of the mitochondrial SERAT2;2 transcript was observed for high lighttreated wild type plants (4.4-fold) when compared to wild type control plants (Figure 5B). SERAT2;2 transcript levels in leaves of serat2;1 (3.2-fold), cyp20-3.1 (3.1-fold), and cyp20-3.2 (1.9-fold) plants were also significantly increased (Figure 5B). Only wild type (1.8-fold) and cyp20-3.1 (1.7-fold) plants but not serat2;1 


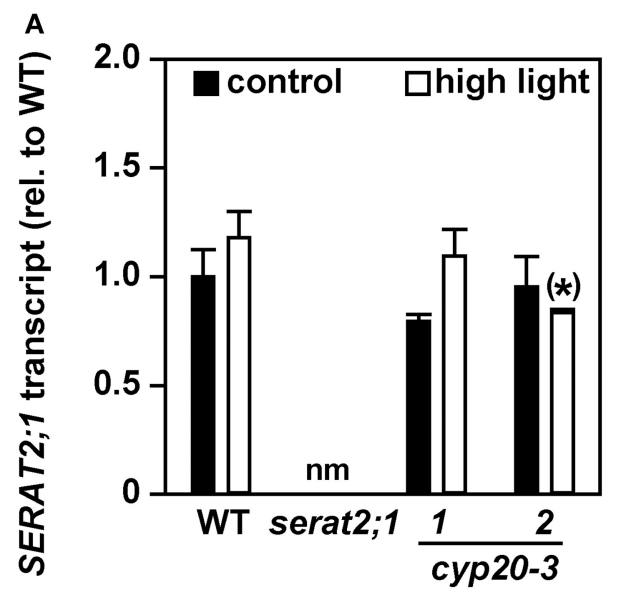

B

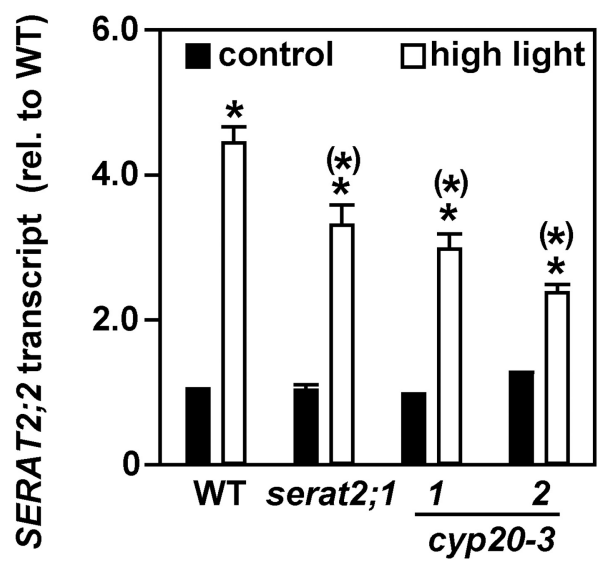

C

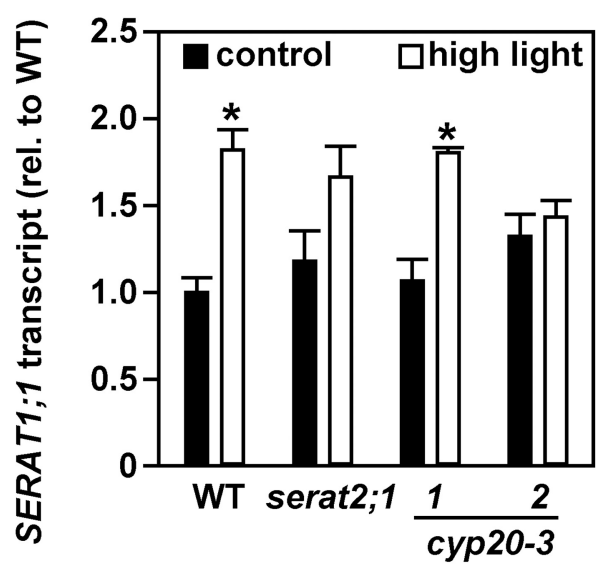

FIGURE 5 | Effect of high light (10 days) on SERAT transcript levels in leaves of wild type (WT) and serat2;1, cyp20-3.1, and cyp20-3.2 mutant lines. SERAT gene expression levels were determined by qRT-PCR in WT and mutant plants grown under control and high light stress conditions. (A) SERAT2;1 transcripts. (B) SERAT2;2 transcripts. (C) SERAT1;1 transcripts. Black bars indicate control conditions, whereas white bars outline stress conditions. Control WT transcript levels were set to $100 \%$. Results represent means $\pm S E(n=3)$. Asterisks indicate statistically significant differences between control and high light treatment ( $p \leq 0.05$; Student's $t$-test). Asterisks in parentheses point to the comparison with high light treated WT ( $p \leq 0.05$; Student's $t$-test) and cyp20-3.2 lines showed a significant increase in SERAT1;1 transcript amount (Figure 5C). These expression analyses suggest that mitochondria and possibly to some extent the cytosol provide most of the SERAT activity at least on this intermediate time scale of a 10 day high light treatment for the enhanced synthesis of OAS and ultimately glutathione during high light acclimation to achieve stress mitigation and growth acceleration. The chloroplasts contribute to this process and improve it but are dispensable, at least under the conditions tested.

\section{DISCUSSION}

Earlier analyses of cysteine synthesis in Arabidopsis revealed that under regular growth conditions the chloroplasts provide the substrate sulfide, the mitochondria most of the substrate OAS and the cytosol synthesizes most of the cysteine in leaves, but that all compartments in principle are able to produce cysteine (Haas et al., 2008; Heeg et al., 2008; Watanabe et al., 2008a; Birke et al., 2013). This surprising finding raised the question if there is a specific function of cysteine synthesis in chloroplasts under oxidative stress, since significant upregulation of SERAT2;1 gene expression was observed after transfer of the CATALASE2 deficient mutant cat2 from high to ambient $\mathrm{CO}_{2}$ concentrations and concomitant $\mathrm{H}_{2} \mathrm{O}_{2}$ stress (Queval et al., 2009) and directly upon $\mathrm{H}_{2} \mathrm{O}_{2}$ exposure (Genevestigator ${ }^{\circledR} \mathrm{V} 3$; https://genevestigator.com/gv/plant. jsp). In line with this observation a post-transcriptional upregulation of SERAT2;1 activity by the peptidyl-prolyl cis/transisomerase CYP20-3 in the chloroplast stroma was linked to resistance against abiotic stress (Dominguez-Solis et al., 2008) and against biotic stress (Park et al., 2013). While a relatively low light intensity which hardly should be considered as stressinducing for Arabidopsis $\left(300 \mu \mathrm{mol} \mathrm{m}^{-2} \mathrm{~s}^{-1}\right.$ ) (Dominguez-Solis et al., 2008) or uncontrolled greenhouse conditions (Cai et al., 2008) had been applied earlier, the controlled acclimation to $800 \mu \mathrm{mol} \mathrm{m} \mathrm{m}^{-2} \mathrm{~s}^{-1}$ of wild type and mutant plants, either deficient in chloroplast SERAT or strongly depleted of its activation partner CYP20-3 in two mutant alleles, was used as an improved approach to challenge chloroplast cysteine synthesis and indicate a role for this process.

When grown under high light the purple-colored rosette leaves of all genotypes indicated similar anthocyanin accumulation. This confirmed the stress-inducing conditions, since increased anthocyanin levels are considered to reflect stress response to enhanced accumulation of reductive power and carbohydrates (Chalker-Scott, 1999; Vanderauwera et al., 2005; Zeng et al., 2010) and to contribute to scavenging of ROS that are produced in high light-treated plants (Gould et al., 2002). In addition, the glutathione concentration increased in wild type leaves, confirming enhanced demand for cysteine synthesis. This observation was backed up by enriched OAS concentrations, whereas cysteine concentrations remained unchanged in control and high light-treated wild type and mutant plants. This unchanged steady-state might be explained by draining cysteine away to meet the increased demand for glutathione to function as a reductant. This would correspond to the metabolic concept of cysteine as a compound with high turnover but low and rarely changing cellular concentrations (see Hell and Wirtz, 2011, for review). Glutathione levels in turn are known to respond to increasing light intensity but to 
reach a plateau at $100-200 \mu \mathrm{mol} \mathrm{m}^{-2} \mathrm{~s}^{-1}$ (Ogawa et al., 2004; Alsharafa et al., 2014). Glutathione was furthermore shown to be essential for the vacuolar sequestration of anthocyanins in maize and petunia through glutathione $S$-transferases (Alfenito et al., 1998; Edwards et al., 2000; Xiang et al., 2001), suggesting that some of the glutathione was removed from the cytoplasmic pool and probably degraded.

However, under the high light conditions employed here $\left(800 \mu \mathrm{mol} \mathrm{m}^{-2} \mathrm{~s}^{-1}\right.$ for 10 days) the lack of CYP20-3 in the cyp203.1 mutant or the absence of its activation target SERAT2;1 in the chloroplast had only a small effect on the stress phenotypes. Wild type and mutant plants acclimated after transfer to high light, but the increase in fresh and dry weight of mutants lacking SERAT2;1 or CYP20-3 was about 20-30\% smaller than that of wild type. Interestingly, the increased total SERAT activities and the resulting OAS concentrations in the four genotypes followed very much the same pattern under high light stress. Thus, the fact that serat2;1 and cyp20-3 mutant plants achieve less biomass along with less SERAT activity under high light stress compared to wild type can be attributed to chloroplast SERAT activity. In contrast, Dominguez-Solis et al. (2008) reported an already lowered total SERAT activity in non-stressed cyp20-3.1 plants and no increase under stress conditions. While it should be cautioned that the treatment conditions and age of plants were different ( 2 weeks as compared to 4.5 weeks here), the significant increase of total SERAT activity also in the serat2;1 null mutant demonstrates that the SERAT activities in the other compartments must have responded to the high light treatment.

SERAT2;1 forms the cysteine synthase complex with the OASTL B isoform in the stroma of chloroplasts. However, in addition to OAS-TL B the related protein CS26 also consumes OAS: CS26 is a member of the same family of $\beta$-substituted alanine synthases like the OAS-TLs and catalyzes the formation of S-sulfocysteine from OAS and thiosulfate in the thylakoid lumen. Thus, CS26 and OAS-TL B potentially compete for the chloroplastidic OAS pool. Intriguingly, expression of the CS26 gene increases upon transfer to high light according to public databases (Genevestigator ${ }^{\circledR}$ V3; https://genevestigator.com/gv/plant.jsp). Extended transfer of a CS26 null mutant from short-day to long-day or permanent light $\left(120-160 \mu \mathrm{mol} \mathrm{m} \mathrm{m}^{-2} \mathrm{~s}^{-1}\right)$ was reported to result in growth retardation as a result of disturbed redox processes in the thylakoid lumen (Bermúdez et al., 2010, 2012). The control treatment used here is similar to these experiments by shifting serat2;1 and cyp20-3 mutants from short-day to long-day. The serat2;1 mutant was not affected while both cyp20-3 mutants appeared to grow more slowly, suggesting a possible link to CS26 functions. However, a careful statistical analysis revealed only very weak significance for the growth retardation, at least under the conditions used here. An assessment of photosynthetic parameters of cyp-20-3 mutants as carried out for the cs26 mutant (Bermúdez et al., 2012) might be interesting to investigate redox functions of the system. Indeed, the recovery of photosystem II activity was slower in cyp20-3.1 compared to wild type following photoinhibition by high light treatment (Cai et al., 2008). However, the assumed multiple targets of CYP20-3 reflected by its high abundance (Lippuner et al., 1994; Peltier et al., 2006) makes it difficult to dissect its various functions.
Indeed, expression analysis of the three major SERAT genes showed significant increases of SERAT2;2 mRNA and much less of SERAT1;1. The expression of the SERAT2;1 gene remained unchanged, although regulation could also occur on the transcriptional level: SERAT2;1 transcript levels were reported to be up-regulated upon treatment of Arabidopsis roots with the oxidizing reagent menadione (Lehmann et al., 2009) and upon oxidative stress in the leaves of the catalase-deficient mutant (cat2; Queval et al., 2009). This suggests that the observed increases in total SERAT activity are at least only partially due to activation of SERAT2;1 by CYP20-3 but are complemented by the contributions of the other compartments. In fact, the increase of total SERAT activity in high light-treated wild type plants might be mainly attributed to the induction of SERAT2;2 transcript amounts in these plants. Several lines of evidence support this assumption. The plastidic SERAT loss-of-function mutant demonstrates that SERAT2;1 makes a small contribution to total SERAT activity in leaves (Watanabe et al., 2008b; Figure 3A). In contrast mitochondrial SERAT2;2 represents approximately $80 \%$ of total SERAT activity in Arabidopsis leaves (Watanabe et al., 2008b) and consequently triggers the flux of cysteine synthesis, at least under normal growth conditions (Haas et al., 2008; Wirtz et al., 2012).

A specific contribution of CYP20-3 to the formation of the plastidic cysteine synthase complex by its chaperone function may be unnecessary, since formation of the Arabidopsis cysteine synthase complexes is known to occur spontaneously ( $\Delta \mathrm{G}=-33 \mathrm{kcal} \mathrm{mol}^{-1}$ ), without assistance of chaperones (Wirtz et al., 2010) and with an equilibrium dissociation constant of SERAT and OAS-TL subunits of $\mathrm{K}_{\mathrm{D}}=25 \mathrm{nM}$ (Berkowitz et al., 2002). Given the several hundred-fold excess of OASTL over SERAT particularly in chloroplasts of many plant species (reviewed in Hell and Wirtz, 2011), essentially all SERAT molecules can be expected to be bound in the cysteine synthase complex. In addition, measurements of maximal activities of chloroplast SERATs in pea and Arabidopsis indicate only $10-13 \%$ of total SERAT activity in chloroplasts (Ruffet et al., 1995; Watanabe et al., 2008b). However, the function of CYP203 as a mediator of plastidic CSC formation was reinforced by the demonstration of strongly fostered CYP20-3 and SERAT2;1 interaction in the presence of OPDA (Park et al., 2013). In addition, a sequential pull-down assay showed that OPDA stimulates the interaction between SERAT2;1 and plastidic OAS-TL B with CYP20-3 as the mediator (Park et al., 2013). In line with this function the strong phenotypic and biochemical similarities between serat 2;1 and both cyp20-3 mutants provide tentative evidence for a joint and specific mechanism. This indicates that, despite having multiple interaction partners, the activation of total SERAT activity upon high light stress is one of the major contributions of CYP20-3 to cope with this stress. The analysis of a mutant lacking chloroplast OAS-TL B protein (oastlB; Heeg et al., 2008; Birke et al., 2013) under high light conditions could be suitable to test this hypothesis.

Therefore, the following hypothesis is put forward: CYP203 and SERAT1 might contribute to retrograde-signaling of high light stress response instead of direct induction of cysteine synthesis in plastids for enhanced glutathione production. The 
advantage of this mechanism could be the speed of activation, since OPDA was shown to increase rapidly upon exposure to high light (Alsharafa et al., 2014) which could immediately activate chloroplastidic CSC. This scenario does not exclude that CYP20-3 has also additional functions, which are important under nonstress or other stress condition. Thus, it would not be the plastidic SERAT activity per se but the formation of the plastidic CSC itself. CYP20-3 promoted formation of the CSC upon high light might trigger through a yet not characterized signaling cascade the expression of SERAT2;2 (and probably more) transcripts. The mitochondrial OAS might be transported from mitochondria to plastids and to the cytosol as evidenced (Wirtz et al., 2012; Birke et al., 2013; Lee et al., 2013), to generate cysteine to be used for glutathione biosynthesis. Kinetic analysis of OAS, cysteine and glutathione levels with subcellular resolution would have to be developed to validate this model.

\section{MATERIALS AND METHODS PLANT GENOTYPES AND GROWTH UNDER CONTROL AND STRESS CONDITIONS}

All experiments were conducted using Arabidopsis thaliana, ecotype Columbia-0, as the wild type control and T-DNA insertion mutants, which derived from the same background. Seeds of cyp20-3.1 (SALK_001615; AT3G62030) and cyp20-3.2 (SALK_054125; AT3G62030) were obtained from the SALK collection (Salk Institute Genomic Analysis Laboratory) and the serat2;1 knock-out mutant (SALK_099019; AT1G55920) from Watanabe et al. (2008b). cyp20-3.2 plants were tested for homozygosity by PCR after Sambrook et al. (1989) with primers 1587_TTTGGCGAAAACTCTTAGCTG), 1588_TGGATTTAACACAAGCGGTTC and 1401_ATTTTGCC GATTTCGGAAC. Genomic leaf DNA was isolated according to Edwards et al. (1991).

Seeds were stratified on soil for 2 days at $4^{\circ} \mathrm{C}$ and subsequently transferred for germination to growth chambers. Plants were initially grown under short-day conditions with a day/night cycle of $8.5 / 15.5$ h. Humidity was set to $60 \%$ and light intensity to $100 \mu \mathrm{mol} \mathrm{m} \mathrm{m}^{-2} \mathrm{~s}^{-1}$. After 3 weeks the plants were transferred for 10 days to long-day conditions with a day/night cycle of $16 / 8 \mathrm{~h}$ and light intensities of either $800 \mu \mathrm{mol} \mathrm{m} \mathrm{m}^{-2}$ $\mathrm{s}^{-1}$ (high light) or $100 \mu \mathrm{mol} \mathrm{m} \mathrm{m}^{-2} \mathrm{~s}^{-1}$ (low light control). For fresh weight analysis whole rosettes were weighed, for dry weight determination the fresh material was kept for $3-5$ days at $60^{\circ} \mathrm{C}$ beforehand.

\section{ANTHOCYANIN DETERMINATION}

Determination of anthocyanin content was performed based on the spectral characteristics of cyanidin-derived anthocyanins. The procedure was modified after Giusti and Wrolstad (2001) and Gou et al. (2011). Pigments were extracted in $1 \mathrm{ml} 0.1 \% \mathrm{HCl}$ (in ethanol) from $30 \mathrm{mg}$ ground leaf tissue on a platform shaker at $40 \mathrm{rpm}$ overnight at $4^{\circ} \mathrm{C}$. Cell debris was sedimented at $20,000 \times$ $\mathrm{g}$ for $10 \mathrm{~min}$ at $4^{\circ} \mathrm{C}$. Absorbance of the anthocyanin was measured spectrophotometrically at $535 \mathrm{~nm}$ and $700 \mathrm{~nm}$ and anthocyanin concentration was calculated according to Giusti and Wrolstad (2001).

\section{DETERMINATION OF METABOLITES}

Hydrophilic metabolites were extracted from leaves of control and high light-treated Arabidopsis leaves as described in Wirtz and Hell (2003). Thiols and OAS contents were quantified according to Heeg et al. (2008) in cooperation with the Metabolomics Core Technology Platform Heidelberg funded by the DFG Excellence inititative.

\section{DETERMINATION OF SERAT ENZYME ACTIVITY AND IMMUNOLOGICAL DETECTION OF PROTEINS}

SERAT activity, coupled to the OAS-TL reaction, was determined based on spectrophotometrical cysteine detection described by Gaitonde (1967). Total soluble protein extracts were prepared from $200 \mathrm{mg}$ ground leaf tissue according to Birke et al. (2013) using Spintrap G-25 columns (GE Healthcare, München). Subsequently protein concentrations as well as enzymatic activities were determined as described by Heeg et al. (2008). Equal amounts of the crude extract were separated by discontinuous SDS-PAGE and blotted on PVDF membrane using a TransBlot ${ }^{\circledR}$ Cell system (Bio-Rad, München). Immunological detection of CYP20-3 was done using $\alpha$-CYP20-3 in combination with a horseradish peroxidase-conjugated secondary antibody and chemiluminescent detection. Loading of protein was tested by staining the gel with Coomassie Brilliant Blue G-250 (Merck, Darmstadt).

\section{TRANSCRIPT OUANTIFICATION BY qRT-PCR}

Gene expression levels were verified in leaves using the principles of the quantification of cDNA targets with quantitative real-time PCR (qRT-PCR). Total RNA was extracted from $100 \mathrm{mg}$ of Arabidopsis leaf tissue using the peqGOLD Total RNA Kit (Peqlab, Erlangen). cDNA was synthesized using the M-MLV Reverse Transcriptase-Kit (Promega, Mannheim). Transcript amount of the respective genes were determined with the RotorGene SYBRGreen PCR Kit (Qiagen, Hilden) and the Rotor-Gene Q system (Qiagen, Hilden).

Gene specific primers used for qRT-PCR: CYP20-3 (2102 CTGGACCTGGAATCTTGAGC; 2103_CTTGTCCAAACACGA CATGC; 2104_CCACCAAGCATCAGAGAACC; 2105_CCAGCA ACTTCACCTCCAAT); SERAT2;1 (for_CACATGCCGAACCGG TAATAC; rev_GGTGAATCTTCCGGTTTACAGAGA); SERAT2;2 (for_AATGGAACCCAGACCAAAACC; rev_GCCCAAACATCA TCGACTTCA); SERAT1;1 (for_TGGACACAGATCAAGGCGG; rev_ATGAGAAAGAATCGTCGAATATAGATAGC); PP2a_PDF2 (for_CTTCTCGCTCCAGTAATGGGACC; rev_GCTTGGTCGA CTATCGGAATGCGCG)

\section{ACKNOWLEDGMENTS}

The authors are grateful for financial support by the Deutsche Forschungsgemeinschaft to Rüdiger Hell and the Schmeil Foundation Heidelberg to Anna Speiser.

\section{SUPPLEMENTARY MATERIAL}

The Supplementary Material for this article can be found online at: http://www.frontiersin.org/journal/10.3389/fpls.2014. 00776/abstract 


\section{REFERENCES}

Alfenito, M. R., Souer, E., Goodman, C. D., Buell, R., Mol, J., Koes, R., et al. (1998). Functional complementation of anthocyanin sequestration in the vacuole by widely divergent glutathione S-transferases. Plant Cell 10, 1135-1149. doi: 10.1105/tpc.10.7.1135

Alsharafa, K., Vogel, M. O., Oelze, M.-L., Moore, M., Stingl, N., König, K., et al. (2014). Kinetics of retrograde signalling initiation in the high light response of Arabidopsis thaliana. Phil. Trans. R. Soc. B 369, 20130424. doi: 10.1098/rstb.2013.0424

Barber, J., and Andersson, B. (1992). Too much of a good thing: light can be bad for photosynthesis. Trends Biochem. Sci. 17, 61-66. doi: 10.1016/09680004(92)90503-2

Berkowitz, O., Wirtz, M., Wolf, A., Kuhlmann, J., and Hell, R. (2002). Use of biomolecular interaction analysis to elucidate the regulatory mechanism of the cysteine synthase complex from Arabidopsis thaliana. J. Biol. Chem. 277, 30629-30634. doi: 10.1074/jbc.M111632200

Bermúdez, M. Á., Galmés, J., Moreno, I., Mullineaux, P. M., Gotor, C., and Romero, L. C. (2012). Photosynthetic adaptation to length of day is dependent on S-sulfocysteine synthase activity in the thylakoid lumen. Plant Phys. 160, 274-288. doi: 10.1104/pp.112.201491

Bermúdez, M. Á., Paez-Ochoa, M. A., Gotor, C., and Romero, L. C. (2010). Arabidopsis S-sulfocysteine synthase activity is essential for chloroplast function and long-day light-dependent redox control. Plant Cell 22, 403-416. doi: 10.1105/tpc.109.071985

Birke, H., Heeg, C., Wirtz, M., and Hell, R. (2013). Successful fertilization requires the presence of at least one major $O$-Acetylserine(thiol)lyase for cysteine synthesis in pollen of Arabidopsis. Plant Physiol. 163, 959-972. doi: 10.1104/pp.113.221200

Cai, W., Ma, J., Guo, J., and Zhang, L. (2008). Function of ROC4 in the efficient repair of photodamaged photosystem II in Arabidopsis. Photochem. Photobiol. 84, 1343-1348. doi: 10.1111/j.1751-1097.2008.00448.x

Chalker-Scott, L. (1999). Environmental significance of anthocyanins in plant stress responses. Photochem. Photobiol. 70, 1-9. doi: 10.1111/j.1751-1097.1999.tb01944.x

Chan, K. X., Wirtz, M., Phua, S. Y., Estavillo, G. M., and Pogson, B. J. (2013). Balancing metabolites in drought: the sulfur assimilation conundrum. Trends Plant Sci. 18, 18-29. doi: 10.1016/j.tplants.2012.07.005

Chou, I., and Gasser, C. (1997). Characterization of the cyclophilin gene family of Arabidopsis thaliana and phylogenetic analysis of known cyclophilin proteins. Plant Mol. Biol. 35, 873-892. doi: 10.1023/A:1005930024796

Dominguez-Solis, J. R., He, Z., Lima, A., Ting, J., Buchanan, B. B., and Luan, S. (2008). A cyclophilin links redox and light signals to cysteine biosynthesis and stress responses in chloroplasts. Proc. Natl. Acad. Sci. U.S.A. 105, 16386-16391. doi: 10.1073/pnas.0808204105

Eckardt, N. A., Snyder, C. W., Portis, A. R., and Ogren, W. L. (1997). Growth and photosynthesis under high and low irradiance of Arabidopsis thaliana antisense mutants with reduced ribulose-1,5-bisphosphate carboxylase/oxygenase activase content. Plant Physiol. 113, 575-586. doi: 10.1104/pp.113.2.575

Edwards, K., Johnstone, C., and Thompson, C. (1991). A simple and rapid method for the preparation of plant genomic DNA for PCR analysis. Nucleic Acids Res. 19:1349. doi: 10.1093/nar/19.6.1349

Edwards, R., Dixon, D. P., and Walbot, V. (2000). Plant glutathione S-transferases: enzymes with multiple functions in sickness and in health. Trends Plant Sci. 5, 193-198. doi: 10.1016/S1360-1385(00)01601-0

Foyer, C. H., Bloom, A. J., Queval, G., and Noctor, G. (2009). Photorespiratory metabolism: genes, mutants, energetics, and redox signaling. Annu. Rev. Plant Biol. 60, 455-484. doi: 10.1146/annurev.arplant.043008.091948

Foyer, C. H., and Noctor, G. (2011). Ascorbate and glutathione: the heart of the redox hub. Plant Physiol. 155, 2-18. doi: 10.1104/pp.110.167569

Fryer, M. J., Oxborough, K., Mullineaux, P. M., and Baker, N. R. (2002). Imaging of photo-oxidative stress responses in leaves. J. Exp. Bot. 53, 1249-1254. doi: 10.1093/jexbot/53.372.1249

Gaitonde, M. K. (1967). A spectrophotometric method for the direct determination of cysteine in the presence of other naturally occuring amino acids. Biochem. J. 104, 627-633.

Giusti, M. M., and Wrolstad, R. E. (2001). "Characterization and Measurement of Anthocyanins by UV-Visible Spectroscopy," in Current Protocols in Food Analytical Chemistry (Hoboken, NJ: John Wiley \& Sons, Inc. F:F1:F1.2). doi: 10.1002/0471142913.faf0102s00
Gou, J.-Y., Felippes, F. F., Liu, C.-J., Weigel, D., and Wang, J.-W. (2011). Negative regulation of anthocyanin biosynthesis in arabidopsis by a miR156-targeted SPL transcription factor. Plant Cell 23, 1512-1522. doi: 10.1105/tpc.111. 084525

Gould, K. S., McKelvie, J., and Markham, K. R. (2002). Do anthocyanins function as antioxidants in leaves? Imaging of $\mathrm{H}_{2} \mathrm{O}_{2}$ in red and green leaves after mechanical injury. Plant Cell Environ. 25, 1261-1269. doi: 10.1046/j.13653040.2002.00905.x

Grzam, A., Martin, M., Hell, R., and Meyer, A. (2007). $\gamma$-glutamyl transpeptidase GGT4 initiates vacuolar degradation of glutathione $S$-conjugates in Arabidopsis. FEBS Lett. 581, 3131-3138. doi: 10.1016/j.febslet.2007.05.071

Haas, F. H., Heeg, C., Queiroz, R., Bauer, A., Wirtz, M., and Hell, R. (2008). Mitochondrial serine acetyltransferase functions as a pacemaker of cysteine synthesis in plant cells. Plant Physiol. 148, 1055-1067. doi: 10.1104/pp.108.125237

He, Z., Li, L., and Luan, S. (2004). Immunophilins and parvulins. Superfamily of peptidyl prolyl isomerases in Arabidopsis. Plant Physiol. 134, 1248-1267. doi: 10.1104/pp.103.031005

Heeg, C., Kruse, C., Jost, R., Gutensohn, M., Ruppert, T., Wirtz, M., et al. (2008). Analysis of the Arabidopsis $\mathrm{O}$-acetylserine(thiol)lyase gene family demonstrates compartment-specific differences in the regulation of cysteine synthesis. Plant Cell 20, 168-185. doi: 10.1105/tpc.107.056747

Hell, R., and Wirtz, M. (2011). Molecular Biology, Biochemistry and Cellular Physiology of Cysteine Metabolism in Arabidopsis thaliana. Arabidopsis Book 9:e0154. doi: 10.1199/tab.0154

Howarth, J. R., Dominguez-Solis, J. R., Gutierrez-Alcala, G., Wray, J. L., Romero, L. C., and Gotor, C. (2003). The serine acetyltransferase gene family in Arabidopsis thaliana and the regulation of its expression by cadmium. Plant Mol. Biol. 51, 589-598. doi: 10.1023/A:1022349623951

Hruz, T., Laule, O., Szabo, G., Wessendorp, F., Bleuler, S., Oertle, L., et al. (2008). Genevestigator V3: a reference expression database for the metaanalysis of transcriptomes. Adv. Bioinformatics 2008:420747. doi: 10.1155/2008/ 420747

Khan, M. S., Haas, F. H., Allboje Samami, A., Moghaddas Gholami, A., Bauer, A., Fellenberg, K., et al. (2010). Sulfite reductase defines a newly discovered bottleneck for assimilatory sulfate reduction and is essential for growth andd in Arabidopsis thaliana. Plant Cell 22, 1216-1231. doi: 10.1105/tpc.110.074088

Laxa, M., Konig, J., Dietz, K. J., and Kandlbinder, A. (2007). Role of the cysteine residues in Arabidopsis thaliana cyclophilin CYP20-3 in peptidyl-prolyl cis-trans isomerase and redox-related functions. Biochem. J. 401, 287-297. doi: 10.1042/BJ20061092

Lee, C. P., Wirtz, M., and Hell, R. (2013). Evidence for several cysteine transport mechanisms in the mitochondrial membranes of Arabidopsis thaliana. Plant Cell Physiol. 55, 64-73. doi: 10.1093/pcp/pct155

Lehmann, M., Schwarzlander, M., Obata, T., Sirikantaramas, S., Burow, M., Olsen, C. E., et al. (2009). The metabolic response of Arabidopsis roots to oxidative stress is distinct from that of heterotrophic cells in culture and highlights a complex relationship between the levels of transcripts, metabolites, and flux. $\mathrm{Mol}$. Plant 2, 390-406. doi: 10.1093/mp/ssn080

Lippuner, V., Chou, I. T., Scott, S. V., Ettinger, W. F., Theg, S. M., and Gasser, C. S. (1994). Cloning and characterization of chloroplast and cytosolic forms of cyclophilin from Arabidopsis thaliana. J. Biol. Chem. 269, 7863-7868.

Marty, L., Siala, W., Schwarzlander, M., Fricker, M. D., Wirtz, M., Sweetlove, L. J., et al. (2009). The NADPH-dependent thioredoxin system constitutes a functional backup for cytosolic glutathione reductase in Arabidopsis. Proc. Natl. Acad. Sci. U.S.A. 106, 9109-9114. doi: 10.1073/pnas.0900206106

May, M. J., Vernoux, T., Sanchez-Fernandez, R., Van Montagu, M., and Inze, D. (1998). Evidence for posttranscriptional activation of $\gamma$-glutamylcysteine synthetase during plant stress responses. Proc. Natl. Acad. Sci. U.S.A. 95, 12049-12054. doi: 10.1073/pnas.95.20.12049

Meyer, A. J., and Hell, R. (2005). Glutathione homeostasis and redox-regulation by sulfhydryl groups. Photosynth. Res. 86, 435-457. doi: 10.1007/s11120-0058425-1

Motohashi, K., Koyama, F., Nakanishi, Y., Ueoka-Nakanishi, H., and Hisabori, T. (2003). Chloroplast cyclophilin is a target protein of thioredoxin: thiol modulation of peptidyl-prolyl cis-trans isomerase activity. J. Biol. Chem. 278, 31848-31852. doi: 10.1074/jbc.M304258200

Noctor, G., and Foyer, C. H. (1998). Ascorbate and glutathione: keeping active oxygen under control. Annu. Rev. Plant Physiol. Plant Mol. Biol. 49, 249-279. doi: 10.1146/annurev.arplant.49.1.249 
Noctor, G., Mhamdi, A., Chaouch, S., Han, Y. I., Neukermans, J., Marquez-Garcia, B., et al. (2012). Glutathione in plants an integrated overview. Plant, Cell Environ. 35, 454-484. doi: 10.1111/j.1365-3040.2011.02400.x

Noctor, G., Queval, G., Mhamdi, A., Chaouch, S., and Foyer, C. H. (2011). Glutathione. Arabidopsis Book 9, e0142. doi: 10.1199/tab.0142

Ogawa, K., Hatano-Iwasaki, A., Yanagida, M., and Iwabuchi, M. (2004). Level of glutathione is regulated by ATP-dependent ligation of glutamate and cysteine through photosynthesis in Arabidopsis thaliana: mechanism of strong interaction of light intensity with flowering. Plant Cell Physiol. 45, 1-8. doi: $10.1093 / \mathrm{pcp} / \mathrm{pch} 008$

Park, S.-W., Li, W., Viehhauser, A., He, B., Kim, S., Nilsson, A. K., et al. (2013). Cyclophilin 20-3 relays a 12-oxo-phytodienoic acid signal during stress responsive regulation of cellular redox homeostasis. Proc. Natl. Acad. Sci. U.S.A. 110, 9559-9564. doi: 10.1073/pnas.1218872110

Peltier, J.-B., Cai, Y., Sun, Q., Zabrouskov, V., Giacomelli, L., Rudella, A., et al. (2006). The oligomeric stromal proteome of arabidopsis thaliana chloroplasts. Mol. Cell. Proteomics 5, 114-133. doi: 10.1074/mcp.M500180-MCP200

Queval, G., Thominet, D., Vanacker, H., Miginiac-Maslow, M., Gakiere, B., and Noctor, G. (2009). $\mathrm{H}_{2} \mathrm{O}_{2}$-activated up-regulation of glutathione in Arabidopsis involves induction of genes encoding enzymes involved in cysteine synthesis in the chloroplast. Mol. Plant 2, 344-356. doi: 10.1093/mp/ssp002

Romano, P. G. N., Horton, P., and Gray, J. E. (2004). The Arabidopsis cyclophilin gene family. Plant Physiol. 134, 1268-1282. doi: 10.1104/pp.103. 022160

Rossel, J. B., Walter, P. B., Hendrickson, L., Chow, W. S., Poole, A., Mullineaux, P. M., et al. (2006). A mutation affecting ASCORBATE PEROXIDASE 2 gene expression reveals a link between responses to high light and drought tolerance. Plant, Cell Environ. 29, 269-281. doi: 10.1111/j.1365-3040.2005.01419.x

Rouhier, N., Lemaire, S. D., and Jacquot, J.-P. (2008). The role of glutathione in photosynthetic organisms: emerging functions for glutaredoxins and glutathionylation. Annu. Rev. Plant Biol. 59, 143. doi: 10.1146/annurev.arplant.59.032607.092811

Ruffet, M. L., Lebrun, M., Droux, M., and Douce, R. (1995). Subcellular distribution of serine acetyltransferase from Pisum sativum and characterization of an Arabidopsis thaliana putative cytosolic isoform. Eur. J. Biochem. 227, 500-509.

Sambrook, J., Fritsch, E. F., and Maniatis, T. (1989). Molecular Cloning: A Laboratory Manual. New York, NY: Cold Spring Harbour Laboratory Press.

Suzuki, N., Koussevitzky, S., Mittler, R. O. N., and Miller, G. A. D. (2012). ROS and redox signalling in the response of plants to abiotic stress. Plant, Cell Environ. 35, 259-270. doi: 10.1111/j.1365-3040.2011.02336.x

Szechyńska-Hebda, M., and Karpiński, S. (2013). Light intensity-dependent retrograde signalling in higher plants. J. Plant Physiol. 170, 1501-1516. doi: 10.1016/j.jplph.2013.06.005

Vanderauwera, S., Zimmermann, P., Rombauts, S., Vandenabeele, S., Langebartels, C., Gruissem, W., et al. (2005). Genome-wide analysis of hydrogen peroxideregulated gene expression in Arabidopsis reveals a high light-induced transcrip- tional cluster involved in anthocyanin biosynthesis. Plant Physiol. 139, 806-821. doi: $10.1104 /$ pp. 105.065896

Wang, P., and Heitman, J. (2005). The cyclophilins. Genome Biol. 6:226. doi: 10.1186/gb-2005-6-7-226

Watanabe, M., Kusano, M., Oikawa, A., Fukushima, A., Noji, M., and Saito, K. (2008a). Physiological roles of the $\beta$-substituted alanine synthase gene family in Arabidopsis. Plant Physiol. 146, 310-320. doi: 10.1104/pp.107.106831

Watanabe, M., Mochida, K., Kato, T., Tabata, S., Yoshimoto, N., Noji, M., et al. (2008b). Comparative genomics and reverse genetics analysis reveal indispensable functions of the serine acetyltransferase gene family in Arabidopsis. Plant Cell 20, 2484-2496. doi: 10.1105/tpc.108.060335

Wirtz, M., Beard, K. F. M., Lee, C. P., Boltz, A., Schwarzländer, M., Fuchs, C., et al. (2012). Mitochondrial cysteine synthase Complex regulates $O$ acetylserine biosynthesis in plants. J. Biol. Chem. 287, 27941-27947. doi: 10.1074/jbc.M112.372656

Wirtz, M., Birke, H., Heeg, C., Mueller, C., Hosp, F., Throm, C., et al. (2010). Structure and function of the hetero-oligomeric cysteine synthase complex in plants. J. Biol. Chem. 285, 32810-32817. doi: 10.1074/jbc.M110.157446

Wirtz, M., and Hell, R. (2003). Production of cysteine for bacterial and plant biotechnology: application of cysteine feedback-insensitive isoforms of serine acetyltransferase. Amino Acids 24, 195-203. doi: 10.1007/s00726-002-0313-9

Xiang, C., Werner, B. L., Christensen, E. M., and Oliver, D. J. (2001). The biological functions of glutathione revisited in Arabidopsis transgenic plants with altered glutathione levels. Plant Physiol. 126, 564-574. doi: 10.1104/pp.126.2.564

Zeng, X.-Q., Chow, W. S., Su, L.-J., Peng, X.-X., and Peng, C.-L. (2010). Protective effect of supplemental anthocyanins on Arabidopsis leaves under high light. Physiologia Plantarum 138, 215-225. doi: 10.1111/j.1399-3054.2009. 01316.x

Conflict of Interest Statement: The authors declare that the research was conducted in the absence of any commercial or financial relationships that could be construed as a potential conflict of interest.

Received: 14 November 2014; accepted: 15 December 2014; published online: 21 January 2015.

Citation: Speiser A, Haberland S, Watanabe M, Wirtz M, Dietz K-J, Saito K and Hell $R$ (2015) The significance of cysteine synthesis for acclimation to high light conditions. Front. Plant Sci. 5:776. doi: 10.3389/fpls.2014.00776

This article was submitted to Plant Physiology, a section of the journal Frontiers in Plant Science.

Copyright (C) 2015 Speiser, Haberland, Watanabe, Wirtz, Dietz, Saito and Hell. This is an open-access article distributed under the terms of the Creative Commons Attribution License (CC BY). The use, distribution or reproduction in other forums is permitted, provided the original author(s) or licensor are credited and that the original publication in this journal is cited, in accordance with accepted academic practice. No use, distribution or reproduction is permitted which does not comply with these terms. 